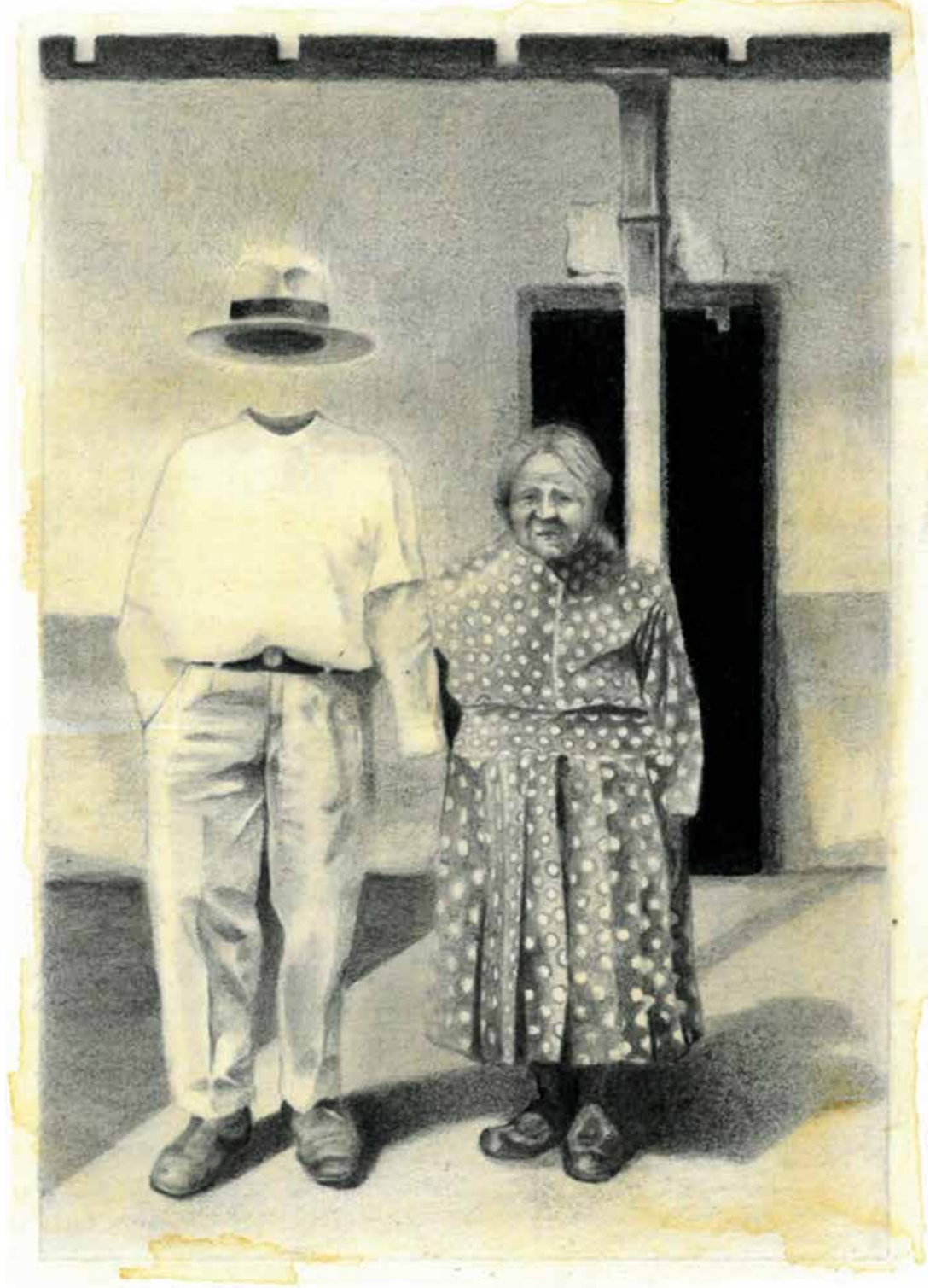

Luis Fernando Salazar Espinosa

Sin título

De la serie Incorpóreo

Lápiz sobre papel

$18 \times 15 \mathrm{~cm}$

2017

Medellín 


\title{
Actores, motivaciones e intereses de la paradiplomacia en la frontera del Caribe insular colombiano después del fallo de La Haya*
}

\author{
Duván Fernando Ramírez Zamora (Colombia)** \\ Silvia Cristina Mantilla Valbuena (Colombia)*** \\ Raúl Román Romero (Colombia)****
}

\section{Resumen}

El artículo estudia la influencia de los intereses y las motivaciones políticas e internacionales del gobierno local y la sociedad civil del departamento colombiano Archipiélago de San Andrés, Providencia y Santa Catalina, en el ejercicio de la paradiplomacia desarrollada en el escenario transfronterizo con el Gran Caribe después de la crisis sociopolítica ocasionada por el fallo de La Haya en 2012. Se realiza un análisis descriptivo y cualitativo del estudio de caso para concluir que, a pesar de compartir objetivos comunes, tanto el gobierno local como la sociedad civil desarrollan estrategias particulares de inserción internacional determinadas por distintos niveles de conflictividad frente al gobierno nacional. Estas fluctúan de una paradiplomacia tradicional basada en el relacionamiento armónico con el accionar internacional y los intereses del Estado colombiano —en el caso del gobierno local-, a tendencias de carácter protodiplomático guiadas por motivaciones fuertemente

\footnotetext{
* Este artículo es el resultado del proyecto de investigación «Las relaciones políticas y diplomáticas de Colombia con los países del Caribe centroamericano» de la Universidad Nacional de Colombia Sede Caribe. Código Hermes: 39549.

** Politólogo. Investigador del grupo Nación, Nación Región y Relaciones Internacionales en América Latina y el Caribe, Universidad Nacional de Colombia. Correo electrónico: dframirezz@unal.edu. co - Orcid: 0000-0002-1066-7648 - Google Scholar: https://scholar.google.com/citations?user=cAvgzgAAAAJ\&hl

*** Politóloga. Magíster en Estudios Latinoamericanos. Doctorada en Migraciones y Conflictos en la Sociedad Global. Docente de la Facultad de Derecho, Ciencias Políticas y Sociales, Universidad Nacional de Colombia Sede Bogotá e investigadora del grupo Nación Región y Relaciones Internacionales en América Latina y el Caribe de la misma institución. Correo electrónico: scmantillav@unal.edu.co Orcid: 0000-0001-5645-3349 - Google Scholar: https://scholar.google.com/citations?hl=es\&user=Y wtEMugAAAAJ

${ }^{* * * *}$ Historiador. Magíster en Estudios del Caribe. Doctorado en Historia de América Latina. Docente de la Universidad Nacional de Colombia Sede Caribe e Investigador del grupo Nación Región y Relaciones Internacionales en América Latina y el Caribe. Correo electrónico: rromanr@unal.edu.co - Orcid: 0000-0002-3186-5168 - Google Scholar: https://scholar.google.com/citations?hl=es\&user=H 8FZ7IEAAAAJ
} 
identitarias, autonomistas e incluso contestatarias —en el caso de la sociedad civil representada por The Archipelago Movement for Ethnic Native Self Determination (AMEN-SD)-.

\title{
Palabras clave
}

Relaciones Internacionales; Paradiplomacia; Protodiplomacia; Fronteras; Gran Caribe; Colombia.

Fecha de recepción: junio de 2019 • Fecha de aprobación: octubre de 2019

\section{Cómo citar este artículo}

Ramírez Zamora, Duván Fernando; Mantilla Valbuena, Silvia Cristina y Román Romero, Raúl. (2020). Actores, motivaciones e intereses de la paradiplomacia en la frontera del Caribe insular colombiano después del fallo de La Haya. Estudios Políticos (Universidad de Antioquia), 57, pp. 42-69. DOI: 10.17533/udea.espo.n57a03

\section{Actors, Motivations, and Interests of the Parallel Diplomacy in the Border of the Colombian Insular Caribbean After the Hague Judgment}

\begin{abstract}
This article studies the influence of varied political and international interests and motivations fostered by the local government and the civil society from the Colombian archipelago of San Andrés, Providencia and Santa Catalina. This research focuses on the exercise of paradiplomatic activities in the cross-border scenario of the Great Caribbean after the sociopolitical crisis caused by the Hague's border ruling in 2012. This case study analysis is based on a descriptive and qualitative approach. It concludes that although both the civil society and the local government may share common goals, each of them uses strategies for international insertion which are determined by numerous levels of political conflict against the national central government. Moreover, these strategies may vary from the traditional paradiplomacy based on harmonious relations towards the international acts and the interest of the Colombian State (local government), to protodiplomatic tendencies guided by strong identitarian, autonomist and even non-conformist motivations (in the case of the civil society represented by The Archipelago Movement for Ethnic Native Self Determination [AMEN-SD] group).
\end{abstract}

\section{Keywords}

International Relations; Paradiplomacy; Protodiplomacy; Boundaries; Great Caribbean; Colombia. 


\section{Introducción}

En las relaciones internacionales (RR. II.) la paradiplomacia es un concepto que se ha venido consolidando ${ }^{1}$ como un nicho analítico sobre la participación y toma de decisiones autónomas de los gobiernos no centrales (GNC) que ocurren en el ámbito internacional de manera paralela al accionar de los Estados (Cornago,2000, p. 56) y que recientemente también está siendo utilizado para describir el comportamiento internacional de otros actores locales como las organizaciones de la sociedad civil.

Esta actividad internacional de los entes locales en los espacios fronterizos configura diferentes formas de interacción en las que progresivamente son suprimidas restricciones políticas, económicas y sociales para propiciar nuevas relaciones e intercambios de carácter «glocal», entendidas estas como aquellas prácticas yuxtapuestas entre los órdenes global y local donde transitan flujos de capitales y se producen acciones de cooperación (Machado y Feitoza, 2018, p. 64). Dichas acciones se traducen luego en estrategias propicias para el desarrollo de relaciones paradiplomáticas que se afianzan en lo territorial y que permiten la negociación de los conflictos y la promoción de las agendas de los actores locales.

A pesar de contar con un espacio multifronterizo en el mar Caribe, en Colombia aún no ha sido concebida la integración de las fronteras marítimas de manera proactiva, debido a que estas zonas carecen de políticas públicas para su internacionalización (Mantilla, Chacón y Román, 2016; Mantilla, 2018) y tampoco existen estudios que sistematicen o evalúen el accionar internacional de los actores locales. ${ }^{2}$ En el caso de San Andrés, la frontera marítima de Colombia en el Caribe revela una serie de tensiones limítrofes, vacíos institucionales y deficiencias estructurales que contrastan con la actividad internacional de carácter autónomo y cada vez más visible de los actores locales para resolver sus demandas o para reforzar la integración transnacional.

\footnotetext{
${ }^{1}$ Los estudios sobre la paradiplomacia de los gobiernos no centrales tienen a las universidades de EE. UU y de Europa como pioneras (Zeraoui, 2013). Aunque el estudio inició desde la década de 1970 se limitó a una descripción de las acciones más que a un debate conceptual, hasta que Ivo Duchacek (1984) propuso el concepto.

${ }^{2}$ El Documento Conpes 3805 del 10 de mayo de 2014, Prosperidad para las fronteras de Colombia, realiza un diagnóstico sobre las principales problemáticas de las fronteras marítimas del país.
} 
En este contexto se percibe un aumento de la participación social en el plano internacional tanto en el caso del gobierno local del departamento archipiélago, como de la comunidad nativa raizal, en especial de The Archipelago Movement for Ethnic Native Self Determination (AMEN-SD), ${ }^{3}$ que será considerado en esta investigación como el representante de la sociedad civil. Esta actividad responde recientemente a las consecuencias políticas, sociales y económicas que supuso la sentencia de la Corte Internacional de Justicia (CIJ) en 2012 sobre el litigio fronterizo entre Colombia y Nicaragua, el cual otorgó a este último país la jurisdicción de 75000 km2 de área marítima en detrimento de los intereses de Colombia (CIJ, 2012, noviembre 19).

Como consecuencia del diferendo, se produjo la vulneración de los derechos territoriales consagrados en los artículos 7. y $13 .^{\circ}$ del Convenio 169 de la Organización Internacional del Trabajo (OIT), el cual creó una sensación de desprotección en la población raizal agravada por la afectación de la pesca artesanal como actividad productiva. Un hecho seriamente cuestionado en la forma en que el Gobierno colombiano planteó la defensa frente al fallo fue no haber permitido la participación de los raizales en los litigios, situación que expresó las históricas diferencias entre los nativos y el Estado nacional acerca de la manera idónea de administrar los recursos marinos, así como sus posibilidades de autonomía y descentralización con el fin de incidir directamente en el devenir de la comunidad.

A partir de lo descrito, este artículo se propone responder a la siguiente pregunta: ¿cómo influyen las motivaciones e intereses del gobierno local y la sociedad civil organizada de la isla de San Andrés en el desarrollo de acciones paradiplomáticas en un contexto de disputas limítrofes y de crisis de relacionamiento del Estado colombiano en el escenario fronterizo del Caribe? Para ello, resulta perentorio evidenciar las características identitarias de las unidades de análisis, en tanto se constituyen en factor determinante de su actuación internacional en un ámbito donde el Estado ha venido redefiniendo sus competencias y delegando diversas funciones a los entes subnacionales, mientras se abre un campo cada vez más amplio a la participación de las organizaciones de la sociedad civil. Por ello es evidente que el GNC del

\footnotetext{
${ }^{3}$ Organización fundada en 1999 y encabezada por pastores bautistas y sacerdotes católicos que abogan por «la protección y promoción de los derechos humanos de la población raizal del archipiélago» (AMEN-SD, 1999, p. 1).
} 
archipiélago y el AMEN-SD tienen particularidades respecto a su identidad y objetivos, a la vez que realizan de formas distintas el ejercicio internacional, siendo para el primero una acción propia de la paradiplomacia tradicional y para el segundo una práctica social, divergente y ampliada de los alcances del concepto que varía según las herramientas institucionales y administrativas.

Metodológicamente, la investigación es de corte cualitativo y se basa en el análisis documental y la aplicación de entrevistas semiestructuradas. Se realizaron catorce entrevistas: a cinco funcionarios del GNC, a dos miembros de AMEN-SD y a siete ciudadanos que han desempeñado cargos diplomáticos o han estado inmiscuidos en las relaciones exteriores del archipiélago y la nación. Lo anterior permitió identificar las acciones a partir de la revisión de diversas fuentes de información del ámbito local, nacional e internacional, como informes, documentos institucionales elaborados por el gobierno central y no central, la prensa y los estatutos de AMEN-SD.

Considerando lo anterior, se plantea la siguiente hipótesis: las acciones paradiplomáticas realizadas por el GNC y la sociedad civil representada por AMEN-SD del departamento de San Andrés, a pesar de la presencia trasversal de la población raizal en sus construcciones identitarias y normativas, son

[ 46 ] movilizadas por diferentes intereses y motivaciones, siendo en el caso del gobierno local una estrategia para insertarse en los mercados de la economía global fundamentada en las directrices de la paradiplomacia convencional que acompaña los objetivos internacionales del Estado-nación colombiano. En el caso del movimiento AMEN SD, este expresa una visión crítica del reconocimiento de la cultura del pueblo raizal en torno a la búsqueda de su autonomía y autodeterminación, así como en la garantía de los derechos humanos, a través de la promoción de la integración e intercambio transfronterizo con las comunidades hermanas del Caribe. En el caso de la sociedad civil, las acciones internacionales se mueven entre la paradiplomacia ciudadana de inclinación identitaria y la protodiplomacia, la cual tiende a contraponerse a los intereses y objetivos del Estado.

\section{Teoría y práctica de las paradiplomacias}

La paradiplomacia, de manera general, se refiere a «aquella tendencia donde los subgobiernos toman acciones autónomas en cuestión de relaciones internacionales» (Zeraoui, 2013, p. 24). Esta es una consecuencia de la 
descentralización político-administrativa que pone especial atención en el prefijo «para-», ya que denota que dicha acción es «paralela o subsidiaria» a las pretensiones del Ejecutivo nacional. En este sentido, el desescalonamiento administrativo del Gobierno central a las entidades locales hace que la actividad paradiplomática sea exclusiva del poder público subnacional departamentos, ciudades o municipalidades-, excluyendo las acciones de organizaciones como grupos de interés o movimientos culturales (Zeraoui, 2016, p. 2). Dicha definición coincide con la de Noé Cornago (2000), quien conceptualiza este tipo de diplomacia como: «la participación de gobiernos no centrales en las relaciones internacionales a través del establecimiento de contactos permanentes o ad hoc con entidades públicas o privadas extranjeras con el propósito de promover diversos aspectos socioeconómicos o culturales, así como cualquier otra dimensión» (p. 56).

A pesar de que los aportes de estos dos autores se presentan como un derrotero teórico capaz de proveer elementos para analizar las motivaciones e intereses en el ejercicio paradiplomático de las unidades de estudio, es indispensable ampliar su alcance para, en primer lugar, evidenciar la relevancia de otros actores tales como agentes de la sociedad civil, mediante lo que se denomina diplomacia ciudadana; y en segundo lugar, profundizar en los planteamientos de la paradiplomacia transfronteriza con el fin de identificar las particularidades de los actores en dicho campo específico de su aplicación. ${ }^{4}$

Por ello, de manera complementaria, se entiende al actor paradiplomático como aquella unidad del sistema internacional, cuya definición es equiparable a la de actor internacional, que acorde con Esther Barbé (2003) - y fundamentada en los aportes de Richard Mansbach, Yale Ferguson y Donald Lampert (1976) y Caterina García Segura (1993) — es aquel que «tiene habilidad para movilizar recursos, alcanzar sus objetivos y tiene cierta autonomía y capacidad para ejercer influencia sobre otros» ( $p$. 135). De este modo, no solo se define al actor a partir de su pertenencia al poder público local, sino por su capacidad para tramitar recursos de manera autónoma y hacer parte de las dinámicas internas de los Estados.

${ }^{4}$ Zeraoui plantea una definición general del término para todos los campos de acción, incluyendo la paradiplomacia transfronteriza (Zeraoui, 2016, p. 5). 
Dichas cualidades permiten identificar al menos tres tipos de actores: a) el GNC; b) los actores interestatales no gubernamentales (AING), los cuales son organizaciones privadas que actúan dentro del Estado, como los partidos políticos o grupos de presión; y c) cualquier conjunto de personas que no hagan parte de la estructura estatal —-sociedad civil—, pero que actúan en el escenario mundial con intereses en materia política, social y económica (Mansbach, Ferguson y Lampert, 1976, citado en Aranda, Ovando y Corder, 2010, p. 43).

Esta ampliación del espectro de participantes hace que las perspectivas sobre la proyección internacional varíen más allá de la delegación de competencias para la consecución de la política exterior del Gobierno central y sean planteadas diversas demandas e intereses. En el caso de los gobiernos subnacionales, estos suelen alinearse con los intereses del Estado, pero surgen excepciones donde la política internacional del actor local no es la expresión a pequeña escala de la política nacional, sino que suele ser disruptiva y antagónica, como la protodiplomacia (Zeraoui, 2016, p. 2), a la vez que tiene por objetivo la búsqueda de legitimidad en la comunidad internacional, valiéndose de un discurso que busca proyectar una imagen negativa del país (Zeraoui, 2009, p. 20).

Esta disyuntiva se encuentra atravesada por la identidad de los actores, quienes configuran una planificación internacional para revindicar factores culturales y sociales, a la par de conseguir beneficios políticos que refuercen su nacionalismo y la necesidad de proyectarse al exterior como una entidad con intereses propios (Paquin, 2004). Dicha condición identitaria es territorializada, en tanto debe generar un vínculo afectivo con el territorio y su población al desarrollar un apego por este espacio, es decir, un actor territorializado debe tener «la capacidad para producir espacios mediante la delimitación y la valorización espacial, lo que permite consolidar la identidad como base de toda actividad paradiplomática» (Zeraoui, 2016, p. 29).

De manera complementaria, la paradiplomacia transfronteriza se desenvuelve en un escenario de «actuación conjunta de actores tanto públicos como privados, a ambos lados de la frontera» (Rhi-Sausi y Oddone, 2009, p. 37), con el fin de «acercar a los pueblos segmentados para posibilitar la intensificación de lazos en diversos temas» (Fernández, 2005, p. 70). Este alcance transnacional desde las fronteras marítimas se caracteriza por los 
términos geográficos, de distancia y la insularidad, que para el archipiélago sanandresano implica una reducida superficie y la dependencia económica de ciertos productos, haciendo de esta la suma de notas geográficas y socioeconómicas que crean una «región ultraperiférica» de trascendencia subestatal (Sobrido-Prieto y Sobrino, 2003, p. 6). La importancia de las entidades subestatales en el contexto de las regiones ultraperiféricas insulares radica en que los archipiélagos son entendidos como un conjunto de lugares que «actúan en concierto» (Baldacchino, 2015, p. 6) a través de una red de articulación que les permiten desarrollar formas de gobierno propias, guiadas tanto por particularidades históricas, culturales y geopolíticas como por su tendencia a la autonomía y descentralización con el Estado nacional del que dependen (2010, p. 5).

La anterior conceptualización permite analizar el caso del Archipiélago de San Andrés en relación con la actividad diplomática de la sociedad civil, considerando dos factores fundamentales: el primero, las particularidades del territorio en el que se desarrollan las dinámicas de relacionamiento paradiplomático debido a la posición geoestratégica y su condición de zona de frontera en el Caribe suroccidental; y el segundo, las motivaciones e intereses de las unidades de análisis dados por los marcos normativos, las características institucionales y la herencia identitaria compartida con una comunidad transnacional.

\section{Dinamismo sociocultural, político y normativo de la frontera colombiana en el Caribe}

Colombia tiene una posición ventajosa en el subcontinente sudamericano al poseer costas sobre dos océanos. Particularmente en el escenario gran caribeño y debido a la ubicación geoestratégica del departamento archipiélago, el país posee un enorme potencial fronterizo al compartir límites con siete naciones, algunas de las cuales se encuentran en disputas limítrofes dada la abundancia de recursos, como reservas pesqueras y petroleras que hacen de esta región un enclave extractivo de suma importancia. La confrontación diplomática más relevante ha sido el diferendo entre Colombia y Nicaragua iniciado en 2001 y resuelto en 2012 por la CIJ, la cual dictaminó una sentencia a favor del último país, otorgándole 75000 km² de área marítima en el mar Caribe sudoccidental que anteriormente figuraban bajo la soberanía colombiana. Esto agudizó las tradicionales tensiones entre el Gobierno colombiano y la población raizal. 


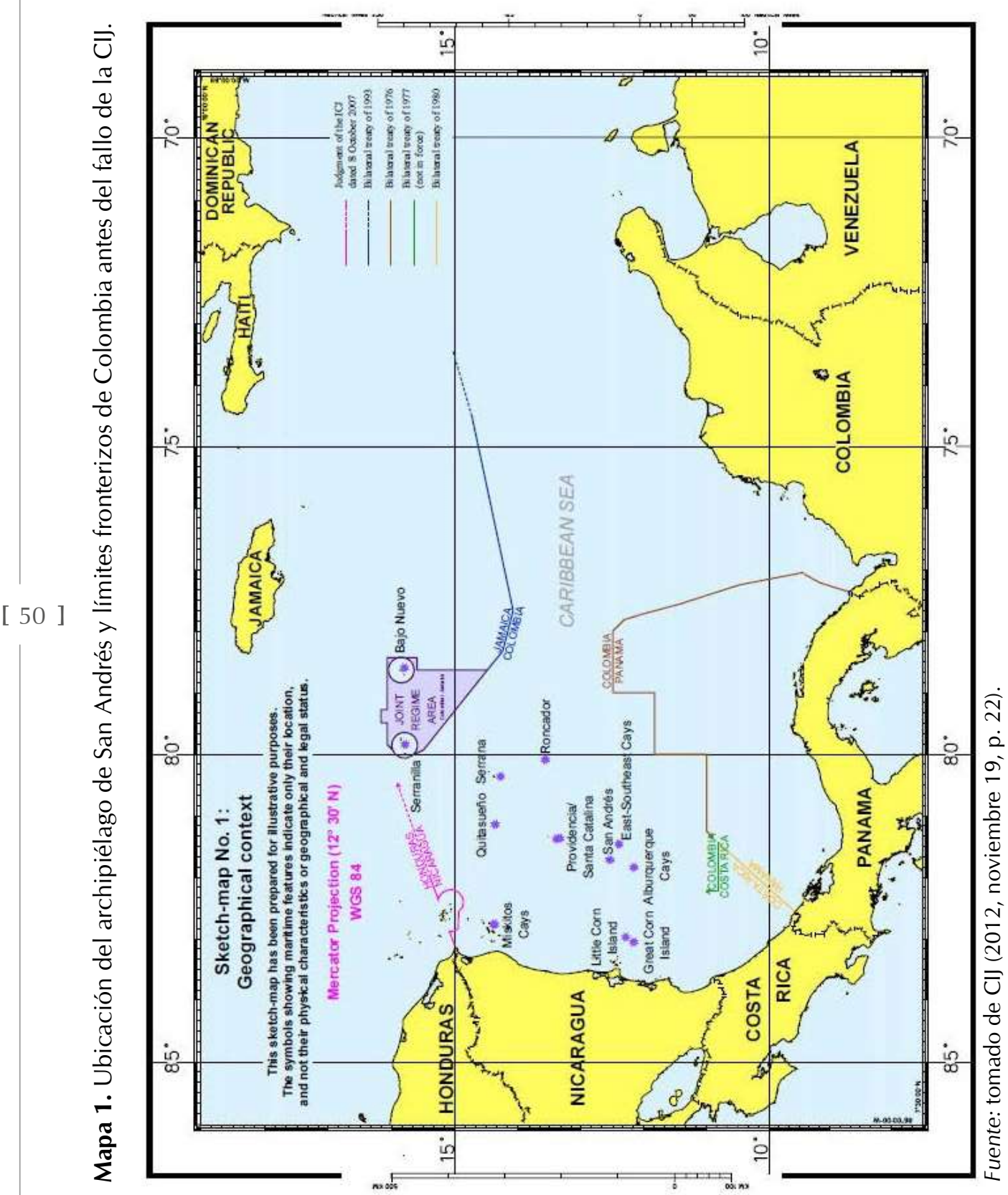


Actores, motivaciones e intereses de la paradiplomacia en la frontera del Caribe insular...

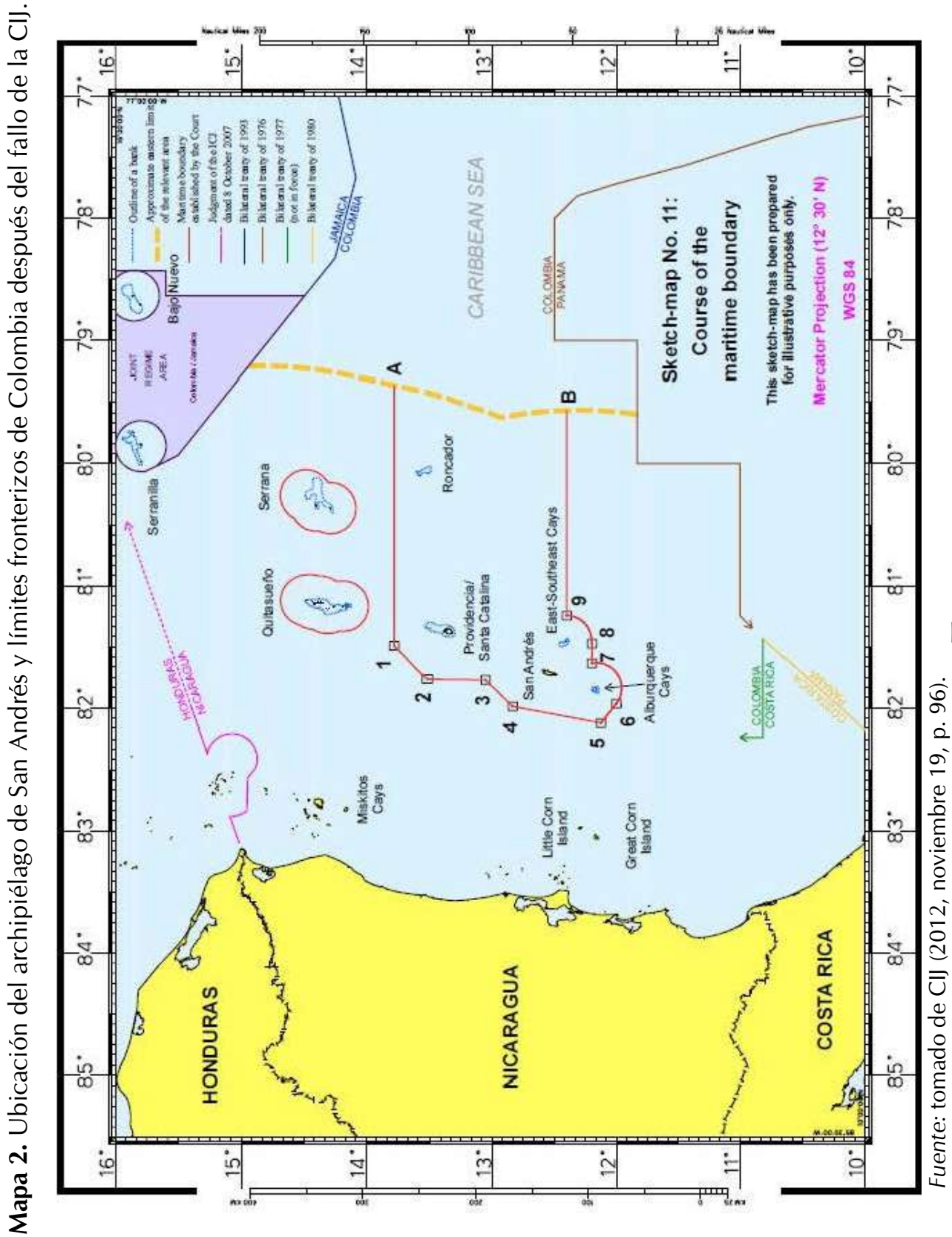


No obstante, la singularidad de la frontera en el Caribe radica en la historia compartida de rasgos socioculturales de las comunidades colindantes del archipiélago de San Andrés, la Región Autónoma de la Costa Caribe sur - Nicaragua - la la provincia de Limón en Costa Rica y Bocas del Toro en Panamá, cuyos orígenes étnicos —afro, especialmente-, religiosos — protestantismo- y lingüísticos —English Caribbean-, ligados a los lazos familiares que han perdurado a pesar de los límites marítimos, contienen las bases para la construcción de un proyecto de integración transfronteriza (Mantilla, Chacón y Román, 2016).

A pesar de lo anterior, se ha evidenciado que las disposiciones normativas nacionales no son suficientes para potenciar tal integración. Aunque el mandato constitucional de 1991 y las posteriores leyes sobre la política exterior y de fronteras han establecido principios generales para su funcionamiento, las prácticas de autogobierno parecen desdibujarse en el escenario de relacionamiento internacional, ya que a pesar de ser contemplada la autonomía de las entidades territoriales dentro de los principios constitucionales del Estado (Constitución Política de Colombia, Art. 1), en realidad predomina un inacabado proceso de descentralización donde las autoridades e instituciones locales carecen de competencias [ 52 ] políticas propias (Sánchez, 2016, p. 45).

En este sentido, desde el marco constitucional, uno de los objetivos esenciales de la política exterior del Estado colombiano es la integración con Latinoamerica y el Caribe con base en principios como la soberanía nacional, la autodeterminación y el reconocimiento del derecho internacional (Constitución Política de Colombia, Art. 9), donde las fronteras se constituyen en un espacio de articulación para el desarrollo de acciones de cooperación con otras naciones, por ejemplo, para la planificación y protección conjunta de ecosistemas naturales situados en zonas fronterizas. De igual forma, en el ámbito subnacional es posible establecer zonas de frontera -terrestres y marítimas - con normas especiales en materia económica y social (Art. 337) donde los gobiernos locales limítrofes podrán adelantar con la entidad territorial colindante del país vecino, de igual ámbito jurisdiccional, programas de cooperación que fomenten el desarrollo comunitario, la prestación de servicios públicos y la preservación del ambiente (Art. 289).

Este marco normativo posiciona a Colombia como uno de los países del Caribe con mayores potencialidades para la consecución de acuerdos 
de integración en diferentes ámbitos (Mantilla y Chacón, 2016), ${ }^{5}$ siendo el caso del archipiélago sanandresano un ejemplo representativo en lo que refiere a la autonomía, la descentralización y la normatividad fronteriza en el ámbito nacional. No obstante, pese a la ventaja geopolítica ${ }^{6}$ que supone la presencia de San Andrés para Colombia en la construcción de lazos comunes transnacionales y las capacidades que otorga la Constitución y la Ley 191 de 1995 para el accionar internacional de los gobiernos subnacionales en contextos fronterizos, no se ha consolidado aún una política integral de largo plazo hacia la región, debido especialmente a que la mencionada ley sobre fronteras, según el Artículo 53, no ha sido aplicada en el contexto del Caribe.

Esta ausencia de directrices sobre el devenir de las fronteras marítimas limita la capacidad de relacionamiento internacional de las unidades de análisis que, no obstante, busca ser subsanada a partir del Documento Conpes ${ }^{7}$ 3805 de 2014 sobre la prosperidad para las fronteras de Colombia, cuyo objetivo es fomentar el desarrollo sostenible mediante el aprovechamiento de potencialidades endógenas y el fortalecimiento institucional, la reducción de las brechas socioeconómicas, la promoción del crecimiento, la integración de estas zonas con el interior del país y los países vecinos y el fortalecimiento de la identidad de los grupos étnicos de frontera (pp. 50-53).

Dicho documento establece trece departamentos que tienen límites terrestres o marítimos con otros países, siendo el archipiélago de San Andrés incluído en una de las Comisiones Regionales para el Desarrollo de las Fronteras (Documento Conpes 3805 de 2014, p. 56). Esta categorización hace que el GNC pueda ejercer las atribuciones constitucionales (Constitución Política de Colombia, Arts. 289 y 337) junto con la gestión del desarrollo regional y las relaciones fronterizas con los pares de países vecinos, así como la formulación de iniciativas que tengan impacto en su territorio, la profundización de la descentralización, la coordinación de la cooperación y el intercambio de información y mercancías (p. 58).

\footnotetext{
${ }^{5}$ En este artículo se demuestra, mediante un balance comparativo de la normatividad fronteriza de cuatro países caribeños, que son justamente Colombia y Nicaragua quienes más desarrollos han logrado en la materia.

${ }^{6}$ Rudolf Kjellén, según Jorge Atencio (1982), definió geopolítica como la «ciencia que concibe al Estado en relación con el espacio que ocupa y los factores económicos y sociales que lo componen» (p. 23).

${ }^{7}$ El Consejo Nacional de Política Económica y Social (Conpes) es un organismo de planeación asesor del Gobierno colombiano sobre temas de desarrollo económico y social. Su objetivo es estudiar y aprobar proyectos de políticas públicas.
} 
A pesar de este primer intento de regulación, el GNC del archipiélago no tiene claramente definidas sus funciones institucionales y los alcances de su accionar en el plano internacional en lo que concierne al relacionamiento con sus pares, no obstante, esto no ha impedido que las administraciones locales hayan desarrollado paulatinamente su actividad diplomática para enfrentar sus propios retos, donde igualmente es perentorio señalar que ni la normativa actual ni la política pública han considerado el papel de organizaciones de la sociedad civil, ya que no existe una referencia tácita sobre sus competencias. Lo anterior contrasta con la evidencia empírica que demuestra las múltiples interacciones con otros actores que trascienden el ámbito subnacional y el activo rol de organizaciones de la sociedad civil.

\section{Configuración identitaria de los actores en la paradiplomacia del departamento Archipiélago de San Andrés, Providencia y Santa Catalina}

La identidad de los actores en relaciones internacionales es un campo aún en discusión, pero Stéphane Paquin (2004) ya introdujo tal categoría alrededor del concepto de paradiplomacia identitaria, según la cual, los objetivos de los actores varían entre el reconocimiento internacional y simbólico de la nación, y el desarrollo local mediante la promoción económica, comercial y la movilidad de personas. Asimismo, a partir del marco analítico del constructivismo ${ }^{8}$ se sostiene que las identidades se construyen a partir de la orientación común de los actores que se encuentran condicionados por estructuras heredadas o por un contexto particular desde donde se interactúa con otras identidades en un campo de intercambios heterogéneo. Por ello, la identidad de los actores en relación con el Estado-nación como la principal figura del sistema internacional puede mutar hacia «arriba» —en unidades supranacionales — o hacia «abajo» —en unidades subnacionales(Merke, 2007, p. 9); también mediante normas constitutivas —formales o informales-, instituciones y modelos cognitivos que son resultado de un determinado proceso histórico.

\subsection{Configuración identitaria del gobierno no central}

Para el GNC, la actividad paradiplomática resulta en una expresión «desde abajo» de su propia identidad, aunque vinculada al fundamento de la legitimidad soberana, la seguridad y la integración del Estado colombiano

${ }^{8}$ Esta investigación no pretende desarrollar un análisis constructivista de la paradiplomacia, sin embargo, se retoman algunos de sus postulados que permiten abordar la identidad de los actores. 
con el Gran Caribe. Por lo tanto, la organización y el funcionamiento del gobierno subnacional en el ámbito internacional depende únicamente de las disposiciones regulatorias de la Constitución Política, de los documentos de política pública como el Conpes 3805 y la Ley 47 de 1993, por las cuales se dictan normas especiales para su ordenamiento administrativo.

Estas disposiciones fortalecen la estructura geopolítica de la frontera, al garantizarle al departamento archipiélago las funciones de coordinación y complementariedad con la nación, a través de herramientas diplomáticas como la capacidad de adelantar acuerdos internacionales con sus pares (Ley 47, Art. 4, núm. 7). De igual manera, este no desconoce su faceta colectiva y las condiciones sociales fronterizas, puesto que, de forma general, aboga por la conservación y promoción de la cultura raizal mediante la protección de su patrimonio (Art. 4, núm. 10). A partir de ello, los artículos 309 y 310 de la Constitución y el artículo 5 de la Ley 47 erigen como régimen departamental especial a San Andrés, Providencia y Santa Catalina en términos administrativos, de comercio exterior y de fomento.

Asimismo, el Departamento estableció su estructura administrativa (Decreto 0227 de 2012) a partir de quince secretarías: Privada, Planeación, Hacienda, Salud, Educación, Agricultura y Pesca, General, Gobierno, Desarrollo Social,Movilidad, Turismo, Infraestructura,ServiciosPúblicos, DeporteyCultura. Ello sin crear una dependencia de relaciones internacionales, aunque desde 2013 funciona la Oficina de Cooperación Internacional adscrita a la Secretaría de Planeación, la cual se encarga, según uno de sus funcionarios (comunicación personal, octubre 14, 2018), de canalizar las convocatorias de la Agencia Presidencial de Cooperación Internacional de Colombia (APC-Colombia). Adicionalmente, otras cuatro secretarías tienen funciones internacionales.

Cuadro 1. Competencias internacionales de las Secretarías Departamentales.

\begin{tabular}{|l|l|}
\hline \multicolumn{1}{|c|}{ Secretaría } & \multicolumn{1}{c|}{ Competencia } \\
\hline Desarrollo Social & $\begin{array}{l}\text { Ejecutar la política social en coordinación con entidades } \\
\text { nacionales e internacionales. }\end{array}$ \\
\hline Educación & $\begin{array}{l}\text { Crear alianzas nacionales e internacionales para la prestación del } \\
\text { servicio educativo. }\end{array}$ \\
\hline Secretaría General & Gestionar recursos de cooperación internacional. \\
\hline Turismo & Gestionar recursos ante entidades internacionales. \\
\hline
\end{tabular}

Fuente: elaboración propia a partir del Decreto Departamental 0227 de 2012. 
Evidenciadas las condiciones institucionales de la identidad del GNC como entidad administrativa estatal, la identificación de las entidades que definen las preferencias nacionales e internacionales resulta compleja debido a que el contexto político del archipiélago se ha caracterizado por una constante inestabilidad gubernamental ocasionada por múltiples casos de corrupción y por la destitución de cinco gobernadores en los últimos seis años (El País, 2018, octubre 13). Esto ha hecho que se evidencie una línea discontinua de acciones, aunque en los planes de desarrollo de Aury Guerrero (2012-2015) (Gobernación del Archipiélago de San Andrés, 2012) y Ronald Housni (2016-2019) (Gobernación del Archipiélago de San Andrés, 2016) se haya identificado la relevancia de la comunidad raizal dentro de los componentes del desarrollo.

A raíz de estas condiciones identitarias son perceptibles las primeras motivaciones para el ejercicio paradiplomático por parte de la Gobernación. Por ello, los motivantes que determinan su accionar son: a) la posición geoestratégica en el Caribe; b) la condición de zona de frontera; c) su categoría de régimen departamental especial; y d) la presencia de una comunidad originaria de carácter transnacional que se articula con el Caribe, a saber, el pueblo raizal (Mantilla, Chacón y Román, 2016).

\subsection{Configuración identitaria de The Archipelago Movement for Ethnic Native Self Determination}

Si bien AMEN-SD ha fundamentado su actividad internacional en los componentes identitarios de la cultural raizal, es necesario aclarar que sus acciones y la descomposición de la variable paradiplomática son la consecuencia de los intereses de un movimiento particular, más no del pueblo raizal en su conjunto, el cual se ha manifestado a través de múltiples formas organizativas que, no obstante, pueden converger entre sí.

Así, el movimiento AMEN-SD fundamenta su construcción identitaria en la dimensión colectiva de la comunidad política al reivindicar las instituciones sociales raizales y al autodefinirse como el pueblo nativo del archipiélago que se enmarca en un histórico proceso de «autorreconocimiento de sus miembros respecto a factores culturales, históricos, lingüísticos, religiosos, fundacionales y de hibridación étnica diferentes a los del Estado Nacional colombiano» (Ortiz, 2013, p. 48). Su identidad se basa en la pertenencia a un territorio situado en el Caribe occidental, el uso del creole como lengua principal, 
una población mayoritariamente negra con herencia cultural angloafricana y tradicionalmente cristiana protestante, pero con una notoria influencia católica (Valencia, 2011, p. 6). Para el líder político Fidel Corpus, la identidad raizal es transfronteriza puesto que «tenemos la misma etnia, cultura, idioma y religión que tienen los pueblos circunvecinos» (comunicación personal, octubre 5, 2018).

Esta condición identitaria, los requerimientos de autonomía política, las denuncias sobre la escasez de recursos, ausencia de servicios públicos y la sobrepoblación, propiciaron desacuerdos respecto al proyecto centralista del Estado-nación colombiano, el cual buscó integrar al archipiélago a través de un fuerte proceso de asimilación que desencadenó desde la década de 1980 en el surgimiento de reivindicaciones étnicas y territoriales. La búsqueda de autonomía en momentos críticos del relacionamiento con el Estado colombiano significó, incluso, la reclamación de independencia, que con el tiempo derivó en una legislación que garantizara la autodeterminación como derecho contemplado inicialmente en la Constitución Política (Arts. 7 y 310), sin que esto afectara la soberanía del Estado, pero reconociendo la capacidad del pueblo raizal de determinar su propio destino (Corte Constitucional, Sentencia C-225 de 1995).

En este contexto nació en 1999 AMEN-SD, autodefinida como «la organización por y para la población Raizal, cuyos lineamientos guían las actuaciones de las demás organizaciones Raizales» (García Taylor, 2003, p. 22). El movimiento aboga por la defensa de los derechos humanos de la población raizal del archipiélago, teniendo como objetivos principales «la búsqueda de autodeterminación y de ayuda nacional e internacional en materia de derechos humanos, el respeto a la cultura y la preservación del inglés Criol» (p. 23), donde se propende por el apoyo y la expresión solidaria de otros grupos étnicos y organizaciones de derechos humanos de alcance global.

La identificación de las instituciones sociales crea parámetros dentro de AMEN-SD que denotan una identidad común a la cultura raizal, pero que adquieren un componente formal y organizado mediante los estatutos que determinan sus expectativas, objetivos y estrategias en el plano internacional a pesar de no existir facultades determinadas por la ley. Al respecto, las motivaciones son: a) la condición geopolítica raizal, es decir, su relación con el territorio mediante la construcción histórica de lazos familiares a través de 
una misma lengua y cultura como factores trascendentales en la integración con el Caribe; y b) las denuncias acerca del abandono de los gobiernos centrales, en tanto no han propendido por las necesidades económicas, políticas y ambientales de los nativos (García Taylor, 2003, p. 12).

La identidad de AMEN-SD construye intereses que han girado mayoritariamente en torno a una esfera autonomista que no ha afectado la integridad del Estado colombiano (Corte Constitucional, Sentencia C-225 de 1995), pero que en ciertos momentos históricos ha adquirido rasgos de un movimiento independentista donde, en la acción internacional se presentan, por un lado, una paradiplomacia ciudadana identitaria que podría extrapolarse al análisis de Paquin (2003) para el caso del GNC de Quebec que podría confluir en la idea de «el reforzamiento de una nación minoritaria en un país multinacional» (p. 12), y por el otro, una lógica protodiplomática que busca la separación respecto del Estado, pero que finalmente se ubica en un punto medio con una identidad definida que revindica a la raizalidad dentro de los márgenes estatales. ${ }^{9}$

El fallo de La Haya en 2012 supuso un motivo para que los raizales responsabilizaran al Estado colombiano de la vulneración de sus derechos [ 58 ] territoriales por la pérdida e integridad de una zona de enorme trascendencia para su supervivencia económica. Por ello y con base en el convenio 169 de la OIT de 2014, AMEN-SD manifestó como principal interés la defensa del derecho de tierras y territorios —incluido los marítimos—, la preservación de las actividades económicas tradicionales y el uso de los recursos naturales por el pueblo Raizal (Art. 15), cuya mayor afectación ha sido evidenciada en el sector de la pesca industrial y artesanal.

Este hecho ha sido un punto de inflexión en el ejercicio paradiplomático, a tal punto que representantes de la sociedad civil y de AMEN-SD establecieron canales de diálogo con diferentes instancias de gobierno y de la sociedad civil en los países fronterizos del Caribe, los cuales incluso trascendieron al escenario global, como será comprobado en los siguientes acápites.

\footnotetext{
${ }^{9}$ Muestra de esta dualidad son las declaraciones de autodeterminación del Pueblo Indígena Raizal (2002, abril 28) y de independencia del AMEN-SD (2008, septiembre 19). La primera fue una protesta por las condiciones sanitarias de la zona circundante al relleno de basura, en tanto la proclama independista pretendía denunciar en el escenario internacional la relación de dominación del Estado colombiano y generar presión para un cambio político en el archipiélago.
} 


\section{Las acciones paradiplomáticas en el departamento Archipiélago de San Andrés, Providencia y Santa Catalina}

\subsection{Las acciones paradiplomáticas del gobierno no central}

De 2012 a 2018 el GNC del archipiélago realizó 49 acciones paradiplomáticas, siendo la cooperación internacional al desarrollo el escenario de mayor preminencia. Los Estados nacionales y los GNC fueron socios estratégicos a través de los programas de asistencia técnica de las agencias de cooperación de China e Israel, además de la creación de lazos con gobiernos locales nacionales como Antioquia y Santander, y con organizaciones como la Asociación de Estados Caribeños (AEC), la Agencia Estadounidense para el Desarrollo Internacional (USAID) y Campus Iberus (Gobernación del Archipiélago, 2016, mayo 26).

En la promoción y proyección externa sobresalen las temáticas de cultura y turismo siendo relevantes la organización de la primera asamblea raizal, el encuentro de Mujeres Negras de Latinoamérica (Barraza, 2013, julio 23) y la 68 edición de la Cumbre de Gobernadores, en donde fue suscrito un convenio de hermanamiento con su par de Santander (Gobernación del Archipiélago, 2013, febrero 19). En el caso del turismo se destacó la participación del gobierno local del archipiélago en el Festival Reina Mundial en Quevedo, Ecuador, y en la Feria Internacional de Turismo de América Latina (FIT) en Buenos Aires (El Isleño, 2018, septiembre 29).

Protocolarmente, es notoria la continua relación con la Gobernación de Santander mediante el Primer Encuentro de Cooperación y Hermanamiento (Gobernación del Archipiélago, 2013, octubre 3), así como la recepción de autoridades extranjeras de las Embajadas de Gran Bretaña (Barraza, 2013, enero 17), Holanda y Corea del Sur (Barraza, 2014, septiembre 11) con fines de cortesía y búsqueda de inversiones. Por su parte, la dimensión de la cooperación internacional al desarrollo posicionó al archipiélago como beneficiario de asistencia técnica y como participante en capacitaciones y seminarios internacionales con el fin de mejorar la prestación de los servicios públicos, el cuidado del medio ambiente y la productividad, siendo fundamental el rol de las agencias de cooperación internacional de los países desarrollados. 


\subsection{Las acciones paradiplomáticas de The Archipelago Movement for Ethnic Native Self Determination}

En cuanto al movimiento AMEN-SD se identificaron trece acciones, mayoritariamente protocolares, centradas en el ámbito transfronterizo y especialmente en el establecimiento de relaciones con Nicaragua, tanto con el presidente Daniel Ortega como con el GNC y la sociedad civil de la Región Autónoma de la Costa Caribe Sur de Nicaragua (Caracol Radio, 2014, octubre 31) en las ciudades de Bluefields y Laguna de Perlas (Rivas, 2014, marzo 11).

A pesar de la poca cantidad de acciones en comparación con el GNC, AMEN-SD desarrolló un notable ejercicio en la dimensión protocolar, destacándose viajes y recibimiento de autoridades del Caribe, especialmente de Nicaragua, con quienes se logró la firma de dos convenios de cooperación: el primero con el gobierno de Daniel Ortega para permitir la actividad pesquera a la comunidad raizal; ${ }^{10}$ y el segundo con la Región Autónoma para intercambios culturales, religiosos y el abastecimiento de combustibles. Paralelamente, en cooperación con la Gobernación del Archipiélago, fue desarrollado la Emansipieshan Kangres — Semana de la Emancipación- con presencia de representantes de Barbados, Trinidad y Tobago, Jamaica y otras

[ 60 ] naciones y comunidades del Gran Caribe (El Isleño, 2018, julio 24).

El movimiento AMEN-SD también recurrió a organismos especializados de la ONU, como el Alto Comisionado para los Derechos Humanos, la Comisión Interamericana de Derechos Humanos (Newball, 2014, noviembre 22) y la Organización de las Naciones Unidas para la Alimentación y la Agricultura (Vanguardia, 2013, marzo 16) para denunciar los efectos negativos del fallo de La Haya. En 2013, por ejemplo, los líderes del movimiento sostuvieron dos reuniones, una en Ginebra y la otra en Bogotá, con la Alta Comisionada para los Derechos Humanos, Navanethem Pillay (Cancillería, 2013, febrero 5), quien pretendía conocer las inquietudes frente a las consecuencias en materia de derechos humanos derivadas del dictamen de la CIJ.

Todas estas acciones tuvieron como objetivo denunciar al Estado colombiano y menoscabar su imagen y legitimidad en el ámbito internacional, acciones que desde la perspectiva del líder social Enrique Pusey (comunicación personal, julio 30, 2016) adquieren matices protodiplomáticos que radican

\footnotetext{
${ }^{10} \mathrm{Al}$ respecto, Corine Duffis, líder raizal, en 2016 manifestó haber logrado un acuerdo de tránsito y de carnetización de los pescadores artesanales (comunicación personal, julio 29, 2016).
} 
en la errática forma en que Colombia dirigió el diferendo fronterizo, al no haber representado adecuadamente los intereses de la población raizal.

\section{Una mirada comparativa entre el gobierno no central y The Archipelago Movement for Ethnic Native Self Determination}

Una primera diferencia entre el comportamiento paradiplomático del GNC y la sociedad civil del departamento archipiélago responde a la cantidad de acciones ejecutadas por cada uno de ellos en virtud de las disparidades presupuestales y administrativas. En el caso del GNC, la cantidad de acciones ha sido mayor en virtud del acceso a recursos y vínculos con países desarrollados, con el Gobierno central y con las agencias consolidadas de la cooperación internacional como APC-Colombia; mientras que en el caso de AMEN-SD, el presupuesto se ha supeditado al aporte voluntario de sus miembros, además de la imposibilidad para recibir cooperación internacional al desarrollo, el cual es un recurso primordialmente intergubernamental. No obstante, AMEN-SD adquiere un rol más diplomático al afianzar su posición en la región a través de la preservación de lazos culturales no solo con sus pares de la sociedad civil, sino trascendiendo al espacio gubernamental mediante diálogos con diferentes GNC, a la vez que posiciona el trámite de demandas y la búsqueda de reconocimiento y apoyo internacional en instancias de carácter intergubernamental como la ONU.

El cuadro 2 resume los tipos de socios y aliados que tuvo cada uno durante el proceso de internacionalización, relaciones que expresan la capacidad que tienen para establecer interacciones con diversos sujetos y escenarios del sistema.

Cuadro 2. Socios externos del gobierno no central de San Andrés y The Archipelago Movement for Ethnic Native Self Determination.

\begin{tabular}{|l|l|l|}
\hline \multicolumn{1}{|c|}{ Socio } & \multicolumn{1}{|c|}{ Departamento de San Andrés } & \multicolumn{1}{c|}{ AMEN-SD } \\
\hline $\begin{array}{l}\text { Gobierno no } \\
\text { central }\end{array}$ & $\begin{array}{l}\text { - Siquirres, Costa Rica } \\
\text { (municipalidad) } \\
\text { - Santander, Colombia } \\
\text { (departamento) } \\
\text { - Antioquia, Colombia } \\
\text { (departamento) }\end{array}$ & $\begin{array}{l}\bullet \text { Región Autónoma Costa Caribe } \\
\text { Sur, Nicaragua (provincia) } \\
\bullet \text { Bluefields, Nicaragua (ciudad) } \\
\bullet \text { Corn Island, Nicaragua } \\
\text { (archipiélago) } \\
\bullet \text { Laguna de Perlas, Nicaragua } \\
\text { (ciudad) }\end{array}$ \\
\hline
\end{tabular}


Cuadro 2. (Continuación)

\begin{tabular}{|c|c|c|}
\hline Socio & Departamento de San Andrés & AMEN-SD \\
\hline Estado & $\begin{array}{l}\text { - Israel } \\
\text { - China } \\
\text { - Japón } \\
\text { - Turquía }\end{array}$ & - Nicaragua \\
\hline $\begin{array}{l}\text { Organizaciones } \\
\text { internacionales }\end{array}$ & $\begin{array}{l}\text { - Agencia de cooperación } \\
\text { internacional de Israel (MASHAV) } \\
\text { - Agencia de cooperación } \\
\text { internacional de Corea } \\
\text { - Agencia de cooperación } \\
\text { internacional de Singapur } \\
\text { - Agencia Española de Cooperación } \\
\text { Internacional para el Desarrollo } \\
\text { (AECID) } \\
\text { - Asociación de Estados Caribeños } \\
\text { (AEC) } \\
\text { - Organización de las Naciones } \\
\text { Unidas para la Alimentación y la } \\
\text { Agricultura (FAO) } \\
\text { - Agencia de los Estados Unidos } \\
\text { para el Desarrollo Internacional } \\
\text { (USAID) }\end{array}$ & $\begin{array}{l}\text { - Oficina del Alto Comisionado } \\
\text { de las Naciones Unidas para los } \\
\text { Derechos Humanos (OACDH) } \\
\text { - Organización de las Naciones } \\
\text { Unidas para la Alimentación y la } \\
\text { Agricultura (FAO) } \\
\text { - Consejo de Derechos Humanos, } \\
\text { Oficina del Alto Comisionado } \\
\text { de las Naciones Unidas para los } \\
\text { Derechos Humanos (OHCHR) } \\
\text { - Secretaria de la Organización } \\
\text { Internacional del Trabajo (OIT) } \\
\text { - Organización de los Estados } \\
\text { Americanos (OEA), Comisión } \\
\text { Interamericana de Derechos } \\
\text { Humanos (CIDH) }\end{array}$ \\
\hline Red & - Onward Internacional & \\
\hline Otros & $\begin{array}{l}\text { - Campus Iberus } \\
\text { - Cancillería de Colombia } \\
\text { - Agencia Presidencial de } \\
\text { Cooperación Internacional de } \\
\text { Colombia (APC-Colombia) }\end{array}$ & $\begin{array}{l}\text { - Cancillería de Colombia } \\
\text { - Bluefields Indian \& Caribbean } \\
\text { University (BICU), Nicaragua }\end{array}$ \\
\hline
\end{tabular}

Fuente: elaboración propia.

En cuanto a sus motivaciones, la diferencia radica, básicamente, en que para el movimiento AMEN-SD el objetivo se centra en «la autonomía del pueblo raizal para tomar decisiones, evitar la pérdida del territorio y luchar por nuestros derechos» (Corinne Duffis, líder raizal, comunicación personal, agosto 29, 2018), mientras que para la gobernación se trata de «fomentar la cooperación y promoción de proyectos que posicionen al departamento» (Rosibel Roa, funcionaria Secretaría de Planeación San Andrés, comunicación personal, octubre 14, 2018). Sin embargo, para Harold Bush, doctor en Historia de las Relaciones Internacionales y Periodista de la Isla (comunicación 
personal, julio 30, 2016), la cooperación del GNC adquiere matices de dependencia y dificultades en la proyección regional, ya que en los planes de desarrollo no ha sido considerada dentro de un proyecto de integración más amplio. A la par, existe una baja complementariedad con los objetivos de AMEN-SD que impide una mayor correlación entre la identidad raizal y el gobierno del archipiélago. Se percibe, asimismo, la falta de interés tanto estatal como de algunos grupos sociales que se expresan, según la líder raizal Corinne Duffis (comunicación personal, agosto 29, 2018) en el hecho de que «no hemos podido avanzar por la falta de recursos y por el poco interés que el Gobierno colombiano tiene en que haya una relación entre la Costa de Mosquitos y los raizales». Por su parte, el político y diplomático raizal Kent Francis (comunicación personal, agosto 30, 2018), considera que la defensa de la pesca tradicional es un punto de comunión entre pueblos que no ha sido aprovechado por la sociedad raizal sanandresana, ya que «no hemos sido capaces de darle suficiente valor a ese contenido».

Finalmente, para Harold Bush (comunicación personal, julio 30, 2016) las dificultades en la internacionalización del grupo AMEN-SD se derivan de su excesiva dependencia de la relación con Nicaragua y en la limitada visión para profundizar diálogos con otros países y comunidades del Caribe. En el caso del GNC, las limitaciones derivan de la imposibilidad para ejercer los instrumentos establecidos en la ley, debido a un generalizado desinterés para crear una secretaría de asuntos raizales.

Se identificaron, no obstante, cuatro aspectosque comparten las unidades de estudio en torno a su acción paradiplomática: a) que están territorializadas identitariamente en el mismo espacio geográfico; b) que debido a su posición geoestratégica en el Caribe, el relacionamiento internacional adquiere mayor relevancia; c) que sus identidades se han configurado en un proceso identitario y cultural de carácter insular que es escencialmente distinto de la identidad central del Estado nacional; y d) que comparten oportunidades y experiencias fácticas y normativas para la cooperación entre unidades, y entre estas y los agentes externos.

\section{Conclusiones}

El estudio de la paradiplomacia vinculada al factor de la identidad territorial es inherente a la proyección internacional de entidades autónomas 
con intereses definidos y separados de los del Estado central. Debido a sus características geopolíticas y por la delegación de atribuciones administrativas contenidas en las disposiciones legales, se identificaron en el caso de la acción paradiplomática del GNC una serie de intereses a largo plazo, como la búsqueda constante de integración mediante la cooperación internacional, suscripción de acuerdos, gestión de programas comunes y creación de alianzas que propendan por el desarrollo institucional, y el fomento económico, comercial y turístico. Esto hace que su accionar gire en torno a las directrices de la paradiplomacia convencional mediante la coordinación con dependencias adscritas al Gobierno central como APC-Colombia, como estrategia para potenciar su inmersión en las dinámicas de la economía global.

En cuanto al movimiento AMEN-SD, las motivaciones fueron, en primer lugar, la relación sociocultural con su entorno fronterizo; y segundo, las constantes denuncias acerca del abandono de los gobiernos centrales respecto a la población isleña. Esto ha posicionado intereses como la búsqueda de autonomía y autodeterminación, la garantía de los derechos humanos y la promoción de la integración e intercambio con otras comunidades del Caribe. Así, la identidad de AMEN-SD construye intereses que varían entre el logro de una mayor autonomía, sin que esto afecte la integridad del Estado paradiplomacia ciudadana identitaria—, o la consecución de independencia, como el caso de la declaración de 2007 —protodiplomacia—.

Los puntos que diferencian a las unidades de análisis se evidencian especialmente en los recursos y mecanismos que utilizan para la consecución de sus intereses. Mientras el GNC fundamenta sus atribuciones internacionales y las funciones administrativas descentralizadas en la ley y las instituciones establecidas por el Estado central, el movimiento AMEN-SD se basa en instituciones sociales construidas por prácticas comunes sostenidas en una misma cultura, etnia e idioma que les permite incidir fuertemente en la vida pública de la isla.

Por último, la actividad transfronteriza del movimiento AMEN-SD es uno de los múltiples ejemplos en el ámbito mundial de actores transfronterizos en regiones ultraperiféricas que deben ser la base para la reconceptualización de la paradiplomacia en escenarios que, como en el caso del departamento archipiélago, carece de modelos normativos que regulen administrativamente los procesos de negociación, cooperación o integración. 
Actores, motivaciones e intereses de la paradiplomacia en la frontera del Caribe insular...

\section{Referencias bibliográficas}

1. Aranda, Gilberto; Ovando, Cristian y Corder, Alejandro. (2010). Experiencias paradiplomáticas en la región de Tarapacá y su proyección subregional. Estudios Internacionales, 42 (165), pp. 33-74. DOI: 10.5354/0719-3769.2010.12650

2. Archipiélago de San Andrés, Providencia y Santa Catalina. Departamento. Decreto 0227. (29, agosto, 2012). Estructura orgánica de la administración central de la Gobernación del Departamento Archipiélago. San Andrés Isla.

3. Atencio, Jorge. (1982). Que es geopolítica. Buenos Aires: Pleamar.

4. Baldacchino, Godfrey (ed). (2015). More than Island Tourism: Branding, Marketing and Logistic in Archipelago Tourist Destinations. In: Archipelago Tourism: Policies and Practices (pp. 1-18). London: Routledge.

5. Barbé, Esther. (2003). Relaciones internacionales. Madrid: Tecnos.

6. Barraza, Carlos. (2013, enero 17). Visita diplomáticos de embajada de Gran Bretaña a Coral Palace. Gobernación del Archipiélago. Recuperado de https:// sanandres.gov.co/index.php/prensa/enterese/noticias/151-rotador-de-noticias/1049visita-diplomaticos-de-embajada-de-gran-bretana-a-coral-palace

7.I Barraza, Carlos. (2013, julio 23). San Andrés sede del próximo encuentro de mujeres Afro latinoamericanas. Gobernación del Archipiélago. Recuperado de https://www.sanandres.gov.co/index.php/prensa/enterese/noticias/151-rotador-denoticias/1410-san-andres-se-trajo-la-sede-del-proximo-encuentro-de-mujeres-afrolatinoamericanas-2014

8. Barraza, Carlos. (2014, septiembre 11). Ministro Consejero de Corea del Sur visitó a la Gobernadora Aury Guerrero Bowie. Gobernación del Archipiélago. Recuperado de https://sanandres.gov.co/index.php/prensa/enterese/noticias/151rotador-de-noticias/2282-ministro-consejero-de-corea-del-sur-visito-a-la-gobernadoraaury-guerrero-bowie

9. Cancillería de Colombia. (2013, febrero 5). Miembros de la comunidad raizal de San Andrés, Providencia y Santa Catalina se reúnen con Alta Comisionada de las Naciones Unidas para los Derechos Humanos. Recuperado de https://www. cancilleria.gov.co/newsroom/news/miembros-la-comunidad-raizal-san-andresprovidencia-y-santa-catalina-se-reunen-con

10. Caracol Radio. (2014, octubre 31). Movimiento AMEN de San Andrés firmará acuerdo con pueblos de Nicaragua. Recuperado de https://caracol.com.co/ radio/2014/10/31/regional/1414745460_487255.html

11. Colombia. Asamblea Nacional Constituyente. Constitución Política. (20 de julio de 1991). Recuperado de http://www.secretariasenado.gov.co/senado/basedoc/ constitucion_politica_1991.html

12. Colombia. Congreso de la República. Ley 191. (23, junio, 1995). Por medio de la cual se dictan disposiciones sobre Zonas de Frontera. Diario oficial, Bogotá, D.C. N. 41903. 
13. Colombia. Congreso de la República. Ley 47. (19, febrero, 1993). Por la cual se dictan normas especiales para la organización y el funcionamiento del Departamento de San Andrés, Providencia Y Santa Catalina. Diario oficial, Bogotá, D.C. N. 40763.

14. Colombia. Corte Constitucional. Sentencia C-225. (18 de mayo de 1995). Recuperado de https://www.corteconstitucional.gov.co/relatoria/1995/c-225-95.htm

15. Colombia. Departamento Nacional de Planeación. Documento Conpes 3805. (10 de mayo de 2014). Prosperidad para las fronteras de Colombia. Recuperado de https://colaboracion.dnp.gov.co/CDT/Conpes/Econ\%C3\%B3micos/3805.pdf

16. Cornago-Prieto, Noé. (2000). Diplomacia, paradiplomacia y redefinición de la seguridad mundial: dimensiones de conflicto y cooperación. En: Keating, Michael y Aldecoa Luzárraga, Francisco (coords.). Paradiplomacia: las relaciones internacionales de las regiones (pp. 55-78). Barcelona: Marcial Pons.

17. Corte Internacional de Justicia (CIJ). (2012, noviembre 19). Controversia territorial y marítima (Nicaragua c. Colombia). Sentencia. Cancillería de Colombia. Recuperado de https://www.cancilleria.gov.co/sites/default/files/litigio_nicaragua/ PRINCIPALES\%20DOCUMENTOS/traduccion_esp_sentencia_dē_19_de_ noviembre_de_2012.pdf

18. Duchacek, Ivo. (1984). The International Dimension of Subnational SelfGovernment. The Journal of Federalism, 14 (4), pp. 5-31. DOI: 10.2307/3330188

19. El Isleño. (2018, julio 24). Se desarrolló el «Emansipieshan Kangres». Recuperado de http://www.xn--elisleo-9za.com/index.php?option = com

[ 66 ] content\&view = article\&id = 15750:se-desarrollo-con-exito-el-emansipieshan-kangres \&catid $=54$ :eventos\&ltemid $=102$

20. El Isleño. (2018, septiembre 29). Las islas participan en la FIT Buenos Aires. Recuperado de http://www.xn--elisleo-9za.com/index.php?option =com_content\&vi $\mathrm{ew}=$ article \&id $=16182$ :las-islas-participan-en-la-fit-buenos-aires \&catid $=\overline{5} 1$ :turismo\& Itemid $=80$

21. El País. (2018, octubre 13). Gobernador y exgobernadora de San Andrés fueron enviados a la cárcel. Recuperado de https:/www.elpais.com.co/judicial/ gobernador-y-exgobernadora-de-san-andres-fueron-enviados-a-la-carcel.html

22. Fernández Manjón, Desiderio. (2005). El papel de las regiones en la dinámica fronteriza en Europa. Revista CIDOB d'Afers Internacionals, 69, pp. 61-88.

23. García Segura, Caterina. (1993). La evolución del concepto de actor en la teoría de las relaciones internacionales. Revista de Sociología, 41, pp. 13-32. DOI: 10.5565/rev/papers/v41n0.1694

24. García Taylor, Sally Ann. (2003). Los vínculos internacionales del movimiento raizal durante los años noventa: en busca de autonomía y reconocimiento. [tesis inédita de grado]. Facultad de Ciencia Política y Gobierno, Universidad del Rosario, Bogotá, D. C.

25. Gobernación del Archipiélago de San Andrés, Providencia y Santa Catalina. (2013, octubre 3). Intercambio entre departamentos de San Andrés Islas y Santander 
se centraría en turismo, bilingüismo y desarrollo económico. Recuperado de https:// www.sanandres.gov.co/index.php/prensa/enterese/noticias/152-espanol/1576intercambio-entre-departamentos-de-san-andres-islas-y-santander-se-centraria-enturismo-bilingueismo-y-desarrollo-economico

26. Gobernación del Archipiélago de San Andrés, Providencia y Santa Catalina. (2016, mayo 26). Conferencia Internacional de Emprendimiento. Recuperado de https://sanandres.gov.co/index.php/prensa/enterese/noticias/151-rotador-denoticias/3373-conferencia-internacional-de-emprendimiento

27. Gobernación del Archipiélago de San Andrés, Providencia y Santa Catalina. (2012). Plan de Desarrollo Departamental 2012-2015. «Para tejer un mundo más humano y seguro». Recuperado de https:/www.sanandres.gov.co/images/stories/ documentos/PLAN_DESARROLLO_COMPLETO_2012_2015.pdf

28. Gobernación del Archipiélago de San Andrés, Providencia y Santa Catalina. (2016). Plan de Desarrollo Departamental 2016-2019. «Los que soñamos somos más». Recuperado de https://downloads.ctfassets.net/27p7ivvbl4bs/1dXmtZepAlg0ggwk80 6qQs/f3ec1e47ff9aa396f8167415c8f5c5a7/88_SanAndres_PDT_2016-2019.pdf

29. Gobernación del Archipiélago. (2013, febrero 19). Gobernadores de Santander y San Andrés firman acuerdo de cooperación. Recuperado de https://sanandres.gov. co/index.php/prensa/enterese/noticias/151-rotador-de-noticias/1097-gobernadoresde-santander-y-san-andres-isla-firman-acuerdo-de-cooperacion-y-hermanamiento

30. Machado de Oliveira, Tito y Feitoza do Nascimento, Rainne. (2018). La paradiplomacia y las relaciones de cooperación en las regiones frontera: Brasil, Bolivia y Paraguay. Revista Frontera Norte, 30 (60), pp. 57-84.

31. Mansbach, Richard; Ferguson, Yale \& Lampert, Donald. (1976). The web of world politics: Nonstate Actors in the Global System. New York: Prentice Hall.

32. Mantilla, Silvia y Chacón, Christian. (2016). Balance comparativo de la normatividad sobre fronteras en cuatro países del Caribe. Estudios Fronterizos, 17 (34), pp. 1-20. DOI: 10.21670/ref.2016.34.a01

33. Mantilla, Silvia. (2018). Las relaciones regionales y transfronterizas de Colombia en el Caribe en el siglo xxı. Mundo Amazónico, 9 (2), pp. 133-160. DOI: 10.15446/ma.v9n2.65178

34. Mantilla, Silvia; Chacón, Christian \& Román, Raúl. (2016). Toward Building a Cross-Border Integration Region among Five Caribbean Countries. Frontera Norte, 28 (56), pp. 5-33.

35. Merke, Federico. (2007). Identidad y política exterior en la teoría de las relaciones internacionales. Documento de Trabajo, Instituto de Investigación en Ciencias Sociales, Universidad de El Salvador.

36. Newball, Daniel. (2014, noviembre 22). Liderazgo raizal efectuó importante presencia en Bogotá. The Archipiélago Press. Recuperado de https:// thearchipielagopress.co/liderazgo-raizal-efectuo-importante-presencia-en-bogota/ 
37. Organización Internacional del Trabajo (OIT). (2014). Convenio 169 de la OIT sobre pueblos indígenas y tribales en países independientes. Recuperado de https:// www.ilo.org/wcmsp5/groups/public/---americas/---ro-lima/documents/publication/ wcms_345065.pdf

38. Ortiz Roca, Fady. (2013). La Autodeterminación en el Caribe: el caso del Archipiélago de San Andrés, Providencia y Santa Catalina. [Tesis inédita de maestría]. Instituto de Estudios Caribeños, Universidad Nacional de Colombia, San Andrés Isla.

39. Paquin, Stéphane. (2003). Paradiplomatie identitaire en Catalogne. Québec: Université Laval.

40. Paquin, Stéphane. (2004). Paradiplomatie et relations internacionales: Théorie des stratégies internationales des régions face à la mondialisation. Bruxelles: Peter Lang.

41. Pueblo Indígena Raizal. (2002, abril 28). Declaración de Autodeterminación, San Andrés, Islas. Recuperado de https://www.urosario.edu.co/jurisprudencia/catedraviva-intercultural/Documentos/DeclaracionAutodeterminacionRaizal.pdf

42. Rhi-Sausi, José Luis y Oddone, Nahuel. (2009). Fronteras e integración transfronteriza en el Mercosur. En: Rhi-Sausi, J y Conato, Darío (coords.). Cooperación transfronteriza e integración en América Latina. Roma: IILA y CeSPI.

43. Rivas, Raúl Lenin. (2014, marzo 11). Pueblo Raizal de San Andrés busca estrechar lazos con pueblos caribeños nicaragüenses. El 19. Recuperado de https:// www.el19digital.com/articulos/ver/titulo:16986-pueblo-indigena-raizal-de-san-

[ 68 ] andres-busca-estrechar-lazos-con-pueblos-caribenos-nicaraguenses

44. Sánchez, Arnulfo. (2016). Revisión histórica del proceso de descentralización administrativa y sus restos en Colombia, de 1985 a 2016. [Tesis inédita de maestría]. Facultad de Sociología, Universidad Santo Tomás, Bogotá, D. C.

45. Sobrido-Prieto, Marta y Sobrino Heredia, José Manuel. (2003). Las regiones Ultraperiféricas. Hacienda Canaria, 5, pp. 5-36.

46. The Archipelago Movement for Ethnic Native Self Determination (AMEN-SD). (1999) Estatutos del movimiento étnico raizal, San Andrés isla. Manuscrito inédito.

47. The Archipelago Movement for Ethnic Native Self Determination (AMEN-SD). (2008, septiembre 19). El pueblo Raizal. Bajo régimen de etnocidio, está en vías de extinción. Colectivo de Abogados José Alvear Restrepo. Recuperado de https://www. colectivodeabogados.org/EL-PUEBLO-RAIZAL-BAJO-REGIMEN-DE

48. Valencia, Inge. (2011). Impactos del reconocimiento multicultural en el Archipiélago de San Andrés, Providencia y Santa Catalina. Entre la etnización y el conflicto social. Revista Colombiana de Antropología, 47 (2), pp. 69-95.

49. Vanguardia. (2013, marzo 16). Raizales y pescadores de San Andrés se reunieron con la FAO. Recuperado de https://www.vanguardia.com/colombia/ raizales-y-pescadores-de-san-andres-se-reunieron-con-la-fao-AYVL200347 
Actores, motivaciones e intereses de la paradiplomacia en la frontera del Caribe insular...

50. Zeraoui, Zidane (coord.). (2013). La paradiplomacia y las relaciones internacionales de las regiones. En: Teoría y práctica de la paradiplomacia (pp. 9-43). Puebla: Instituto Tecnológico de Monterrey.

51. Zeraoui, Zidane. (2009), Regionalización y paradiplomacia. La política internacional de las regiones. México, D. F.: Montiel y Soriana.

52. Zeraoui, Zidane. (2016). Para entender la paradiplomacia. Desafíos, 28 (1), pp. 15-34. DOI: 10.12804/revistas.urosario.edu.co/desafios/a.4470 